\title{
TAUX D'ACTUALISATION ET OPTIMUM SECOND
}

\author{
par Jean TIROLE *
}

Décembre 1979

$\mathrm{N}^{\circ} 7925$

* Ecole Nationale des Ponts et Chaussées. Cet article a été écrit principalement lors de mon séjour au CEPREMAP (Octobre 77 - Juin 78). Je remercie cet organisme pour son accueil, et Roger Guesnerie pour ses nombreux conseils et encouragements. Bien entendu, je reste seul responsable pour les éventuelles fautes et maladresses. 
Cet article considère la littérature sur le taux d'actualisation en situation d'optimum second. Après examen des raisons pour lesquelles l'approche en termes d'optimum premier est inadéquate, et des quelques arguments s'y rattachant, distinction est faite entre modèles "de politique optimale" et modèles"de réforme". L'accent est mis sur la notion importante de coût d'opportunité des fonds publics. Celle-ci ne doit pas être confondue avec la notion de coût de rareté des fonds publics, dont cet article donne une présentation rigoureuse.

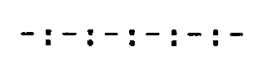

This paper considers the (second best) theory of the social rate of discount. To begin with, the inadequacy of the first best approach and of some related arguments is shown; then the different contributions are divided in "optimal policy models" and "reform models". The important notion of opportunity cost of public funds is emphasized; it should not be confused with the notion of scarcity cost of public funds, whose formalization is given in this paper. 
On sait que l'on peut définir, à partir d'un système intertemporel de prix, une séquence de taux d'intérêt ou d'actualisation égaux aux rapports des prix d'un numéraire d'une période sur I'autre. En tant que sous-produit d'un système de prix, la séquence des taux d'actualisation en conserve les caractéristiques. Elle possède une fonction sélective (orienter l'économie vers les productions et les investissements les plus utiles) et une fonction distributive (agir sur les revenus); en particulier sont en jeu l'allocation entre les secteurs privé et public, l'allocation au sein de ces secteurs, ainsi que la distribution entre générations et à l'intérieur d'une génération (par le financement et la répartition du produit des investissements).

L'objet de cet article est la définition par la Puissance Publique d'un seuil de rentabilité pour ses investissements (ou taux d'actualisation). La traduction de la théorie "optimum-equilibre" et de ses deux théorèmes fondamentaux en termes intertemporels aurait pu conférer aux taux d'intérêt observés un caractère normatif; il n'en fût rien : En effet, il n'existe ni système complet de marchés à terme, ni marché financier parfait, où tous les consommateurs et producteurs seraient présents et pourraient prêter et emprunter à un taux d'intérêt i indépendant de la somme prêtée ou empruntée, et qui, associée à une prévision parfaite des prix futurs, aurait pu être un substitut pour le système de marchés à terme.

Force est alors de poser le problème en termes d'optimum second, c'està-dire de reconnaitre l'existence, et la durabilité de contraites non-parétiennes. Il existe maintenant une litterature considérable sur ce sujet, mais celle-ci forme malheureusement un ensemble très hétéroclite. Il s'avère tout d'abord que le taux d'actualisation est très sensible à la manière dont les investissements publics sont financés et dont leurs bénéfices sont répartis. Ensuite, comme dans d'autres branches de l'Economie Publique, l'on peut considérer soit une politique d'investissement et de financement optimale, soit un petit déplacement du système économique à partir d'un état initial donné ; il en résulte une distinction entre modèles "de politique optimale", et "de politique de réforme" ; c'est à partir de cette ligne de clivage que nous bâtirons notre "survey". 
Avant d'évoquer les distorsions qui rendent nécessaire l'approche en termes d'optimum second, et d'examiner les salutions considérées dans la littérature, donnons quelques définitions : Le taux de préférence pour le présent (TPP) d'un consommateur est le taux marginal de substitution entre ses consommations de numéraire à $t$ et $(t+1)$, moins un. S'il existe un marché financier parfait, le consommateur égalise son TPP et le taux d'intérêt i, car sinon il pourrait augmenter sa satisfaction en prêtant du numéraire (si $i>$ TPP), ou en empruntant (si $i<$ TPP). De même, les producteurs égalisent la productivité marginale de leur capital et le taux d'intérêt afin de maximiser leur profit intertemporel. Enfin le taux social de préférence pour le présent (TSPP) est défini pour la collectivité de façon analogue au TPP pour un consommateur. Dans les conditions d'application des deux théorèmes fondamentaux de l'économie du bien-être, le TSPP n'est autre que le TPP des différents consommateurs, ou la productivité marginale du capital des différentes entreprises.

Une simple observation des taux en vigueur montre que ceux-ci présentent des écarts trop importants pour être dus à des primes de risque (cf ci-dessous). L'on estime à $10 \%$ le taux de productivité marginale pour le secteur privé, alors que le taux d'intérêt net pour les épargnants oscille autour de zéro. Avant d'évoquer les distorsions sous-jacentes à ce phénomène, nous voudrions insister sur le fait que mêmé en leur absence, le TSPP puisse différer des taux lalors communs] de substitution et de transformation intertemporels. C'est l'important argument d'externalités intertemporelles, qui s'analyse comme une divergence entre valeurs individuelles et sociales, et que nous commerçons par rappeler. Nous investiguerons ensuite l'hypothèse de marché financier parfait, condition nécessaire (mais non suffisante) de réalisation décentralisée d'un optimum premier.

\section{$\left.1^{\circ}\right)$ Externalités intertemporelles et préférence sociale pour le présent.}

L'on peut en fait concevoir que la préférence pour le présent des individus et celle de la collectivité divergent, et ce pour trois raisons :

- attitude tutélaire de l'Etat : celui-ci peut juger que l'individu n'est pas en mesure de ou n'a pas l'information pour exercer ses propres choix intertemporels; l'idée est que les agents feraient preuve de "myopie" vis-à-vis de leur propre futur. 
- épargne comme bien public : une insuffisance d'épargne pourrait être aussi dûe à un "phénomène d'isolement" : les individus préfèreraient bénéficier de l'effet externe que représente l'effort d'épargne de leurs contemporains pour assurer le bien être des générations futures plutôt que d'épargner eux-mêmes (problème du "passager clandestin").

- équité intergénérationnelle : la collectivité peut enfin affirmer n'avoir pas de préférence entre les générations, et donc défendre les intérêts des générations futures non représentées face à une insuffisance d'épargne de la génération actuelie.

Nous discuterons ici les deux derniers arguments, dont le point commun est l'accent mis sur l'équité intergénérationnelle. Formellement cependant, le problème de l'épargne comme bien public s'analyse comme un problème d'externalités intragénérationnelles, dans la mesure où la décision d'épargne d’un agent de l'actuelle génération agît sur le bien-être des agents de sa propre génération; la motivation de l'étude toutefois est dans les externalités intergénérationnelles engendrées par les décisions de la génération actuelle. L'argument d'équité intergénérationnelle revient, lui, à conférer à l'Etat un rôle tutélaire vis-à-vis de la génération actuelle, non pour myopie face à son propre futur, mais parce qu'elle. est jugée se comporter "égoistement" en n'épargnant pas assez pour satisfaire les besoins des générations futures non représentées.

\subsection{L'épargne comme bien public.}

Nous étudions ici les théories de Marglin (27a) et Sen (33).

Pour ces auteurs, les membres de la génération actuelle se soucient du bien-être de leurs contemporains, de leurs descendants directs et des descendants de Jeurs contemporains. Pour assurer le bien-être de ces deux dernières catégories, ils adoptent un comportement différent selon qu'ils agissent individuellement ou collectivement. La raison en est qu'ils peuvent bénéficier individuellement d'un effet externe de la part de leurs contemporains quand ceux-ci épargnent. Donnons la présentation simple suivante (due à Sen (33)) : 
Imaginons une génération de $\mathrm{n}$ individus identiques, ayant chacun un descendant direct (l'on considère donc une économie à deux périodes) ; la fonction d'utilité d'un individu représentatif de la première génération est : $U\left(c_{i}, c_{c}, C_{h i}, c_{h c}\right)$

où $\quad C_{i}=$ consommation de l'individu $i$

$C_{C} \quad=$ consommation des contemporains de $i$

$C_{h i}=$ consommation des héritiers de $i$

$C_{\text {hc }}=$ consommation des héritiers des contemporains de $i$

on pose : $\frac{\frac{\partial U}{\partial C_{C}}}{\frac{\partial U}{\partial C_{i}}}=\beta, \frac{\frac{\partial U}{\partial C_{h i}}}{\frac{\partial U}{\partial C_{i}}}=\gamma, \frac{\frac{\partial U}{\partial C_{h c}}}{\frac{\partial U}{\partial C_{i}}}=\alpha$

On suppose que $\alpha, \beta, \gamma$ sont positifs (la fonction d'utilité est alors dite altruiste). On suppose également que tous les membres de la deuxième génération profitent également des bénéfices d'un investissement supplémentaire; soit $(1+a)$ la productivité marginale du capital dans l'économie (sans distinction entre secteurs public et privés.

i) stratégie individualiste : si l'individu i épargne une unité supplémentaire, sa satisfactión varie de :

$$
\delta U_{i} \sim(1+a)\left(\frac{\gamma}{n}+\frac{n-1}{n} \alpha\right)-1
$$

Le taux individuel de préférence pour le présent est alors $a_{C}$ tel que : $1+a_{c}=\frac{n}{\gamma+(n-1) \alpha}$

ii) stratégie de coopération : si les n individus épargnent chacun une unité supplémentaire, la satisfaction de l'individu i varie de :

$$
\delta U_{i} \sim(1+a)(\gamma+(n-1) \alpha)-1-(n-1) \beta
$$

Le taux social de préférence pour le présent est alors ag tel que :

$$
1+a g=\frac{1+(n-1) \beta}{\gamma+(n-1) \alpha} *
$$

* On peut vérifier que cette égalité n'est autre que la condition de Samuelson pour biens publics. 
L'on voit que si $\beta<1$ (une unité de consommation supplémentaire pour $i$ est préférée,par lui à une unité de consommation supplémentaire pour un de ses contemporains), le taux social est inférieur au taux individuel. Pour Marglin (27), la prise en compte des externalités nécessitera donc la réalisation d'investissements de rentabilité trop faible pour être réalisés par le secteur privé (l'Etat peut alors soit pratiquer une politique fiscale et monétaire adéquate, soit réaliser lui-même ces investissements, en tenant compte toutefois du "coût d'opportunité des fonds publics" of 6 ).

A partir de ce modèle, l'on peut faire différentes hypothèses sur la proportion qui revient aux héritiers directs, ou sur les valeurs respectives de $\gamma$ et $a .$. Il nous parait préférable de discuter l'idée fondamentale de ce modèle simple d'épargne comme bien public. Plusieurs critiques ont été adressées à ce modèle :

- pour Tullock (36), puis pour Baumol (6), l'Etat, s'il convenait de cet argument, défavoriserait une génération (actuelle) au profit de générations (futures) sans doute plus aisées (se comportant ainsi à l'encontre de Robin des Bois). Cette critique est quelque peu injustifiée dans la mesure où Sen (33) a fait observer que la richesse des générations futures influe sur $\alpha$ et $\gamma$, mais ne madifie pas les conclusions de notre modèle. Marglin (27) tient également compte de la richesse relative des générations, lorsqu'il énonce que le fondement de la préférence sociale pour le présent est la décroissance de l'utilité marginale*.

- Baumol (7) a fait observer que pour financer les investissements devenus rentables du fait de la prise en compte des externalités, un certain nombre de projets privés seraient supprimés. La critique est également injustifiée dans la mesure où Marglin dans un autre article (27c) a utilisé le concept de coût d'opportunité des fonds publics pour tenir compte de l'effet (en général nocif) du financement de l'investissement public sur l'investissement privé.

$$
. /
$$

* A ce propos, voir aussi feldstein (16) : La donnée d'une fonction d'utilité et de la séquence des consommations futures permettent de calculer la séquence des taux sociaux : $\frac{\frac{\partial U}{\partial c} t}{\frac{\partial U}{\partial c}}-1$ à condition de supposer que l'investissement public influe peu sur la séquence des consommations (sinon les déterminations de la quantité d'investissements publics et du taux de préférence pour le présent doivent être simultanées]. 
- Delorme (11) a montré que les conclusions du Marglin et Sen dependaient fortement du "degré d'altruisme".

\subsection{Equité intergénérationnelle.}

Il se peut que les membres de la génération actuelle ne fassent pas preuve d'altruisme vis-à-vis des générations futures. Même en cas de coopération entre ses membres, la génération actuelle investira relativement peu ; cela tient au fait que les membres des générations futures ne sont pas représentés, ou même ne sont pas nés. L’a Puissance Publique peut estimer qu'elle n'a pas à préférer telle ou telle génération, et en conséquence peut juger que le libre jeu du marché entraine une situation de sous-investissement : c'est l'argument important d'équité intergénérationnelle.

Par rapport aux externalités intragénérationnelles, où l'Etat s'efforçait de résoudre un problème de bien public, les externalités intergénérationnelles conduisent donc à une attitude tutélaire de la Puissance Publique. Les hypothèses sur le degré d'altruisme et la problèmatique sont différentes, mais le résultat est le même : l'Etat juge que l'investissement actuel est insuffisant, et prend des mesures pour y remédier.

\subsection{Implications : Le problème d'externalités ne se pose par définition pas dans} les modèles où tous les agents participent à des marchés à termes, distordus ou non (voir section 7). Il est fondamental dans les autres modèles. Lorsqu'on considère une modification márginale d'une politique d'investissements publics donnée (modèles de réforme), il est possible de fonder la préférence sociale pour le présent sur la décroissance de l'utilité marginale. Le cas des modèles de craissance pose un problème plus complexe : prendre comme fondement unique de la préférence pour le présent la décroissance de l'utilité marginale rend en général impossible la convergence de la fonction objectif quand l'horizon temporel est infini, comme l'a montré Koopmans (25). La solution classique à ce dilemne est l'introduction d'un taux pur $\rho$ de préférence pour le présent de sorte que l'utilité de la consommation à l'époque $t$ est actualisée par le facteur : $e^{-\rho t}$. 


\section{$\left.2^{\circ}\right)$ L'absence de caractère normatif du taux d'intérêt.}

Dans cette section nous évoquons les différentes distorsions qui font que le taux d'intérêt observé (en fait les multiples taux...) n'est pas un indicateur correct d'allocation intertemporelle des ressources.

2.1 Du côté des producteurs : trois groupes d'arguments ont été avancés afin d'expliquer la divergence entre le taux d'intérêt et la productivité marginale des entreprises :

i) Un premier ensemble d'arguments présume de la part de l'entreprise un comportement de non-maximisation du profit (motivations diverses de la "technostructure", tels la maximisation de la croissance ou du bien-être des cadres, etc...).

ii) L'Etat, par son action fiscale, distord les choix de l'entreprise. L'exemple de l'impôt sur les sociétés est bien connu. Pour qu'un investissement, dont les bénéfices sont taxés au taux $50 \%$, soit accepté, il faut que sa productivité soit au moins égale à deux fois le taux d'intérêt. Le coût réel du capital est en fait affecté par d'autres facteurs, tels que la déductibilité de l'intérêt, le traitement de l'amortissement, etc...

iii) Enfin un phénomène important est l'existence de "primes de risque" (approximativement majoration du taux d'actualisation de l'entreprisel liées à l'absence de marchés d'assurance, et très variables selon les secteurs et la taille de l'entreprise. Présentons très brièvement le noeud du problème : Supposons que pour un investisseur ayant de l'aversion pour le risque, un projet de rentabilité $10 \%$ (calculée sur la base de l'espérance mathématique des coûts et bénéfices) soit équivalent à un projet "sûr" de rentabilité $6 \%$. Faisant fi de toutes autres considérations, la collectivité doit-elle entreprendre un investissement (public) de rentabilité $8 \%$ ?

- On peut avancer au nom de l'efficacité de la production globale que I'Etat ne devrait pas réaliser ce projet, qui impliquerait un déplacement de ressources du secteur privé vers des emplois moins rentables (en espérance mathématiquel dans le secteur public. C'est en particulier la position de Hirschleifer (29). Notons que cette position est tout à fait cohérente avec sa recommandation sur le taux d'actualisation (cf 4). De même qu'Harberger (jusqu'en 1968), il suppose que la Puissance Publique a les moyens de corriger les distorsions afin d'imposer les choix intertemporels de la collectivité. Ici le problème consistant à éliminer l'existence de primes de risque dans le secteur privé est difficilement soluble : il semble dangereux de créer un système d'assurances pour la raison simple que les risques de l'entreprise ne sont pas entièrement extérieurs à elle , mais dépendent de son comportement (problème du "hasard moral"). 
- Il semble alors que l'on doive distinguer "risques individuels" et "risques collectifs" (Arrow - Lind (4) ; cette position est aussi adoptée par Boiteux (9), Baumol (7) et Arrow - Kurz (5)). De par la loi des grands nombres, les projets publics ne présentent en général pas de "risques individuels". Les investissements publics ne doivent donc pas comprendre de prime de risque. Ce n'est pas pour autant que tous les projets publics de rentabilité supérieure au taux de préférence pour le présent des consommateurs (ici $6 \%$ ) doivent être réalisés. Il faut tenir compte des répercussions de leur financement et de leur produit sur l'investissement privé, et ce n'est que la résolution du modèle de second rang qui peut nous indiquer le taux d'actualisation approprié.

Etant donnée notre approche en termes d'optimum second, nous nous rallierons à cette deuxième interprétation, tout en gardant bien à l'esprit que les primes de risque du secteur privé sont un facteur important d'écart entre le taux d'intérêt et la productivité marginale du capital privé.

2.2 Du côté des consommateurs : La principale source de distorsion entre le taux d'intérêt et leur TPP semble être l'imposition (impôt sur le revenu, sur les gains en capital, sur I'héritage, etc ....**.

$\left.3^{\circ}\right)$ Financement et produit de l'investissement public.

Dans cette section, nous voudrions aborder deux problèmes envisagés dans la littérature, ayant trait l'un au produit de l'investissement public, l'autre à son financement.

3.1 La notion d' "appropriabilité" (Arrow-Kurz (5)).

L'on dit qu'un investissement public est (totalement) appropriable si la Puissance Publique est en mesure d'exercer une tarification (complète) de ses bénéfices. Il existe plusieurs raisons pour lesquelles un investissement public peut ne pas répondre à cette définition. Tout d'abord, si le marché n'est pas compétitif (ce qui est souvent le cas pour les entreprises publiques), le produit de l'investissement public se divise en deux; une partie monétaire (recettes) et une partie non monétaire (de type surplus). Cette dernière ne peut être appropriée si l'Etat ne peut discriminer entre les différents consommateurs. Ensuite l'appropriabilité peut être techniquement difficile (ou très onéreuse), ou encore n'être pas souhaitable (présence d'effets externes ou de désirs tutélaires:par exemple : l'éducation].

.1.

* Pour une présentation détaillée des effets de l'imposition sur le comportement intertemporel des producteurs et consommateurs, voir Atkinson-Stiglitz (1). 
L'on peut aussi remarquer que si les bénéfices de l'investissement public vont toujours aux consommateurs, la relation peut être directe, ou bien indirecte à travers le processus de production; un certain nombre de projets publics ont en effet pour but d'accrôtre la productivité de la sphère de production privée (exemples : recherche scientifique, irrigation...) tout en pouvant produire aussi des biens de consommation (exemples : autoroutes, éducation...).

En d'autrés termes, les bénéfices des investissements publics peuvent être représentés soit comme des recettes de l'Etat (appropriabilité), soit comme entrant dans la fonction d'utilité des consommateurs, ou encore dans les fonctions de production des entreprises du secteur privé. Plutôt que ses conséquences en termes de distribution, l'effet (supposé néfaste) de la non-appropriabilité d'un investissement public sur l'épargne privée a été abondamment discuté dans la littérature. Feldstein (17) suppose que seuls les coûts et bénéfices monétaires affectent l'épargne privée, tandis que Bradford (10) confère aux éléments non monétaires une influence sur l'épargne privée identique à celle des éléments monétaires.

Ces hypothèses peuvent être fragiles comme l'a montré Bradford ( (10) p.897-898); un parc, une émission de télévision, un programme de sécurité sociale ou une réunion sportive augmentent-ils ou diminuent-ils l'épargne privée ? Et dans quelles proportions?

En tout état de cause, seule la considération du modèle sous-jacent (et en particulier des instruments de politique économiquel permet de déterminer si l'appropriabilité des investissements publics influe sur leur critère de choix*

\subsection{Contraintes budgétaires.}

Certains auteurs à la suite de Boiteux (8) ont supposé que l'une des contraintes non-parétiennes de l'économie prenait la forme d'une contrainte budgétaire rigide. Développant une idée de Thédié (34), nous examinerons dans un cadre intertemporel le rôle de contraintes sur le financement des investissements publics, et nous introduirons les coûts de rareté des fonds publics liés à ces contraintes de financement. Précisons dès maintenant qu'il faut bien distinguer les notions de coût de rareté des fonds publics (cf 7.13), et de coût d'opportunité du capital public (cf 6. .). 
$\left.4^{\circ}\right)$ La nostalgie de l'optimum premier.

Certains arguments ont pour support la théorie de l'optimum premier. Nous avons vu qu'une condition nécessaire de réalisation d'un optimum premier en l'absence d'effets externes est l'égalité des différents taux : de préférence pour le présent et de productivité marginale dans les secteurs public et privé (et du taux d'intérêt si cet optimum premier est obtenu à l'aide d'un marché financier parfait).

4.1 Avec le choix d'un taux d'actualisation public est en fait en jeu le partage des ressources entre les deux secteurs : un faible taux d'actualisation nécessite un drainage de ressources vers le secteur public plus important qu'un fort taux d'actualisation ceci afin de pouvoir financer tous les investissements publics de rentabilité intermédiaire. Il est probable que les méthodes de financement (dette, impôts,...) vont diminuer la quantité de ressources disponibles pour le secteur privé. Certains auteurs ont utilisé l'argument d'efficacité ci-dessus pour demander que la Puissance Publique ne finance pas des projets moins rentables que les projets marginaux du secteur privé : l'Etat doit-il financer des investissements rentables à $5 \%$, quand par ailleurs des investissements privés rentables à $10 \%$ ne peuvent être effectués, faute de fonds ? A priori, il semble qu'une telle opération mènerait à un gaspillage global des ressources, ce qui est confirmé par la théorie de l'optimum premier : les systèmes de prix (et en particulier les taux d'actualisation) doivent être iḍtentiques pour tous les agents.

Ce point de vue est très bien illustré par les écrits d'Harberger jusqu'en 1968 (voir les trois premiers chapitres de (21). Par la suite, Harberger a admis que le coût d'opportunité des fonds publics pouvait differer de la productivité marginale du capital privé : cf. $6^{\circ}$ J. La thèse d'Harberger était alors que, dans les conditions décrites ci-dessus, doivent être acceptés les projets publics ayant un rendement social. d'au moins $10 \%$, et que l'on doit investir les fonds restant disponibles dans le secteur public dans des projets privés ayant une rentabilité de $10 \%$ ou plus. On comprend bien le lien avec la théorie de l'optimum premier : I'on suppose que la Puissance Publique contrôle d'une manière ou d'une autre les investissements du secteur privé, permettant ainsi de lever les obstacles à la réalisation d'un optimum premier. D'autres auteurs, tels que Hirshleifer (22) et Mishan (28), ont adopté des positions assez analogues. 
Le problème fondamental est que la Puissance Publique n'a en général pas accès aux investissements privés. La réalisation d'un optimum premier n'est donc en général pas possible. Il nous faut convenir qu'un certain nombre de distorsions existent dans l'économie, et que la stratégie de la Puissance Publique doit être dictée par la présence de ces distorsions, et non par ce qu'elle devrait faire en leur absence ou si elle disposait d'assez d'instruments pour les corriger, ce qui n'est pas en général le cas. Le fait que le taux marginal de productivité du capital public soit inférieur à celui du capital privé n'implique pas qu'il y ait surinvestissement public : la solution d'optimum second peut exiger un taux plus faible comme nous le verrons.

4.2 Il faut noter que l'argument tiré de la théorie de l'optimum premier n'est pas l'apanage des partisans de la productivité marginale du capital privé comme taux d'actualisation. En effet, cette théorie implique l'égalité du taux d'actualisation non seulement avec la productivité marginale du capital privé, mais aussi avec le taux de préférence pour le présent des consommateurs. Dès lors qu'il y a distorsion entre ces deux derniers taux, l'on peut aussi penser que le taux d'actualisation devrait être le taux de préférence pour le présent des consommateurs, mesuré par le taux d'intérêt (net d'impôt) perçu par eux : c'est la position en particulier de Grjèbine dans un article récent (20).

\section{$\left.5^{\circ}\right)$ Problèmatique de l'optimum second :}

Nous ne discuterons désormais que les contributions des auteurs posant le problème du taux d'actualisation en termes d'optimum second. Nous séparerons leurs apports en deux types d'approches, selon que l'auteur envisage un investissement "marginal" à partir d'un état initial quelconque (réforme) ou bien le calcul de l'optimum. La première approche s'est limitée jusqu'ici (du moins en théorie du taux d'actualisation) au cas où, au mouvement de la production publique correspond une variation des autres paramètres du système économique, ce qui permet d'introduire sans ambiguité la notion de coût d'opportunité du capital public. Cette démarche, qui peut être interprétée en termes de réforme (accroissement local de la fonction objectif ou éventuellement de celle de tous les agents) n'engendre pas forcément une règle de décision menant finalement à un optimum second, la convergence du processus ainsi defini vers un optimum second devant faire l'objet d'une étude particulière. 
Les deux approches traitent d'un même problème : comment le gouvernement doit-il choisir ses investissements quand le secteur privé prend ses décisions d'investissements dans un marché financier comportant des distorsions ? De façon générale, toute variation d'investissement public entre deux états doit être accompagnée par une variation des instruments disponibles (emprunt, impôts...) afin de respecter les contraintes de l'économie. L'on peut dire en simplifiant que les auteurs préconisant l'optimisation acceptent une grande souplesse des instruments, tandis que les partisans de la réforme ne leur permettent que de petites variations. Notons aussi que l'optimalité est un cas particulier de la réforme : en un optimum second, il n'existe pas de variation de l'investissement public et des instruments réalisable et améliorant le critère (la réciproque n'est pas vraie : il peut alors s'agir d'un optimum local).

Arrow et Kurz (5) ont donné la signification suivante à une politique d'investissement optimale : celle-ci consiste à déterminer les décisions optimales à un instant donné, en supposant que les décisions futures seront elles aussi optimales ("an investment policy is a simultaneous choice of present and future investments". p.10]. Cette approche a été vivement critiquée par Harberger, qui estime que le bouleversement radical de la structure fiscale sur le chemin de l'optimum allocatif serait difficile à accepter ((21) ch. V) Il refuse en particulier l'adoption comme taux d'actualisation du taux social de préférence pour le présent, qui, dans le lọng terme du moins, découle du modèle d'Arrow-Kurz . Harberger écrit par exemple ((21) ch.I) : "je crois difficile de soutenir une politique qui imposerait à la communauté des niveaux d'épargne qui seraient nécessaires pour accepter tous les investissements rentables à, par exemple, $4 \%$, et difficile de croire que cela serait possible même si c'était désirable". Par ailleurs, Harberger établit une distinction très nette entre le "Bureau d'évaluation des projets" et le "Ministère des Finances", ce qui semble très critiquable au même titre que l'hypothèse de rigidité totale du système fiscal qu'il emploie dans son modèle (cf 6 ).

La position de Harberger n'est pas générale parmi les partisans de la réforme : Bradford par exemple s'est prononcé en faveur du taux social de préférence pour le présent comme taux d'actualisation, alors qu'il n'est pour Harberger qu'un objectif de long terme, une fois toutes les distorsions éliminées...) 
$\left.6^{\circ}\right)$ Modèles de réforme et coût d'opportunité du capital public.

Nous aurons besoin par la suite de la définition suivante :

Définition : le coût d'opportunité du capital privé à la date $t$ :

$v_{t}$ est la valeur en terme de consommation présente d'une unité supplémentaire (manne céleste) de capital privé à l'époque $t$.

En d'autrés termes, c'est la valeur actualisée à $t$ au taux social de préférence pour le présent du flux de consommation ainsi généré (notons que cela suppose que 1 'on puisse définir de façon non-ambigue les mouvements du système associé à cet apport "extérieur" de capital privé). Ou encore v ${ }_{t}$ unités de consommation à l'époque $t$ sont socialement équivalentes à I unité de capital privé supplémentaire à $t$. Feldstein appelle aussi $v_{t}$ prix fictif du capital privé à $t$.

L'idée fondamentale que l'on retrouve dans tous les modèles de réforme est la suivante : considérons un projet public, et supposons qu'à la réalisation de ce projet soit associé un mouvement et un seul des instruments de politique économique de telle sorte que les contraintes de l'économie restent vérifiées. On peut alors définir de façon non-ambigue deux séquences $\left(--, \delta C_{t},--\right)$ et $\left(--, \delta S_{t},--\right)$ des variations de consommation et de formation de capital privé associées au mouvement du système. On associe à ces deux flux les systèmes de prix fictifs $\left(-\cdots, \pi_{t},--\right)$ et $\left(-\cdots, \pi_{t} y_{t}, \cdots\right)$ où $\pi_{t}=\prod_{\tau=0}^{t-1} \frac{1}{(1+a)^{\tau}}$ est le facteur d'escompte à $t$ associé à la série des taux sociaux de préférence pour le présent.

Considérons maintenant le projet consistant simplement en un financement unitaire à l'époque t.

Définition : le coût d'opportunité des fonds publics $w_{t}$ est la somme des valeurs des deux flux associés à ce projet évalués avec les systèmes de prix fictifs indiqués plus haut*

Chronologiquement, Marglin (27c) fut le premier à développer les implications de ces concepts. Deux contributions importantes furent apportées par Feldstein (17) et Bradford (10). Harberger (21) propose lui une approche légèrement différente, posée en termes de surplus.

* Il va de soi que l'on peut définir de même un coût d'opportunité (ou plutôt une 


\subsection{Marglin (1963 (27c)).}

Marglin envisage un projet comportant un investissement $K(x)$ à l'époque initiale coù $x$ est l'échelle du projet) et un flux de bénéfices nets $\{B(x, t)\}$. Soit a le taux social de préférence pour le présent, supposé constant dans le temps. L'investissement initial est supposé se faire au détriment de $(s k(x))$ unités d’épargne et $((1-s) K(x)$ ) unités de consommation.

i) dans son modèle le plus simple, Marglin suppose qu'une unité supplémentaire de capital privé à $t=0$ produit $r p$ aux dates postérieures et que ce produit est entièrement consommé (pas de réinvestissement) ; rp est appelé productivité marginale du capital privé.

Le coût d'opportunité du capital privé à t=o est alors

$$
v_{0}=\sum_{t=1}^{\infty} \frac{r p}{(1+a)^{t}}=\frac{r p}{a}
$$

Dès lors il est aisé de calculer le coût d'opportunité du capital public :

$$
w_{0}=\frac{r p}{a} s+(1-s)
$$

(puisque la valeur actualisée d'une unité de consommation à l'époque initiale est 1).

Supposons maintenant que les bénéfices nets soient entièrement consommés (ici encore, pas de réinvestissement). Ils ont alors un coût d'opportunité unitaire à chaque période, et le critère de choix de l'investissement est alors :

$$
t=\sum_{=1}^{\infty} \frac{B(x, t)}{(1+a)^{t}}-w_{0} K(x) \geq 0
$$

ii) supposons maintenant qu'une proportion s' des bénéfices nets du projet soit réinvestie dans le secteur privé (et ait donc une rentabilité rp par période), tandis qu'une proportion (1-s') est consommée. Les autres hypothèses étant les mêmes qu'en i), les bénéfices nets ont pour coût d'opportunité (ou plutôt une "valeur d'opportunité")

et le critère devient :

$$
\begin{aligned}
& w_{0}^{\prime}=s^{\prime} \frac{r p}{a}+\left(1-s^{\prime}\right) \\
& w_{0}^{\prime}\left(\sum_{t=1}^{\infty} \frac{B(x, t)}{(1+a)^{t}}\right)-w_{0} K(x) \geq 0
\end{aligned}
$$

Marglin remarque alors que la prise en compte d'un coût d'opportunité des bénéfices nets revient à prendre pour nouveau coût d'opportunité du capital public non pas $w_{0}$, mais $\frac{w_{0}}{w_{0}^{\prime}}=\frac{s r p+(1-s) a}{s^{\top} r p+\left(1-s^{\prime}\right) a}:\left(\sum_{1}^{\infty} \frac{B(x, t)}{\left.(1+a)^{t}\right)}-\frac{w_{0}}{w_{0}^{\prime}} \quad K(x) \geq 0\right.$ 
iii) les modèles I et I' de Marglin (présentés en i) et ii) donnent l'essentiel du raisonnement en termes de coûts d'opportunité. Marglin a complété son étude (modèles II et II') en examinant l'effet du réinvestissement du produit de l'investissement privé (en supposant que celui-ci est réinvesti en proportion s, puis que le produit du réinvestissement est réinvesti en proportion s, etc...).

iv) cette approche a été critiquée par Arrow et Kurz (5) comme étant contraire à une politique d'investissement optimale. Supposons que l'investissement public soit "appropriable" (of 3.1). Supposer que son produit est soit entièrement consommé, soit consommé en proportion s' et investi en proportion (1-s') revient à introduire une rigidité indue dans les allocations futures. Dans un modèle de politique optimale, les allocations futures des bénéfices nets entre consommation privée, investissement privé et investissement public seraient endogènes, sauf contraitre dûment explicitée. Notons que l'allocation des recettes fiscales dans le calcul du coût d'opportunité du capital privé serait sujette à la même critique.

\subsection{Feldstein (1973 (17)) .}

Feldstein a repris et précisé les idées de Marglin, en explicitant le mode de financement (impôt, dette). Un projet public est caractérisé par :

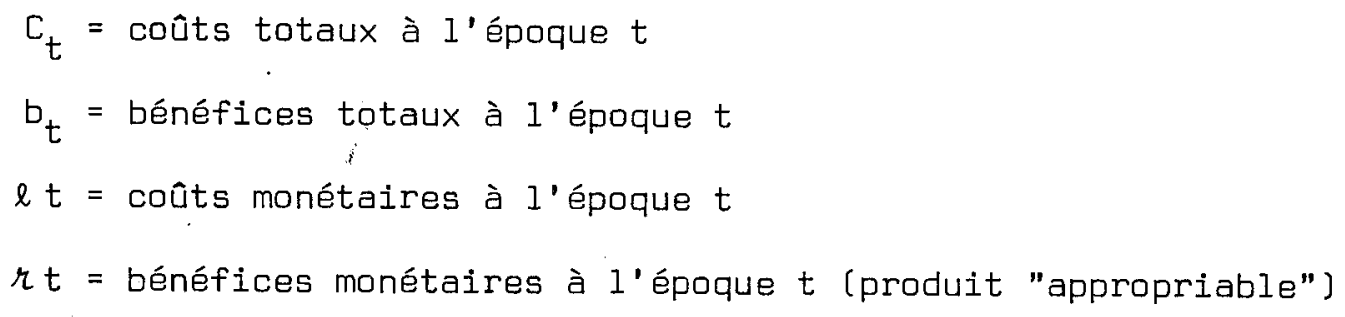

La première hypothèse de Feldstein est de supposer que les éléments non monétaires $\left(C_{t}-\ell_{t}\right)$ et $\left(b_{t}-r_{t}\right)$ n'ont pas d'influence sur l'investissement privé.

La deuxième hypothèse est (comme Marglin) de supposer que $\ell_{t}$ et $r_{t}$ ont une influence symétrique sur l'investissement privé, de sorte que l'on peut ne considérer que leur différence $\left(l_{t}-r_{t}\right)$ (coût monétaire net).

- Si le projet est financé par un impôt (en proportion s $t_{t}$ sur l'investissement privé et $\left(1-s_{t}\right)$ sur la consommation), sa valeur actualisée au taux social de préférence pour le présent est :

$$
V A=\sum_{t=0}^{\infty} \frac{\left(b_{t}-r_{t}\right)-\left(c_{t}-e_{t}\right)-\left(e_{t}-r_{t}\right)\left(s_{t} v_{t}+\left(1-s_{t}\right)\right)}{(1+a) t}
$$


soit encore : $V A=\sum_{t=0}^{\infty} \frac{\left(b_{t}-c_{t}\right)-\left(e_{t}-r_{t}\right) s_{t}\left(v_{t}-1\right)}{(1+a)^{t}}$

- supposons maintenant qu'à $t$ une proportion $\alpha_{t}$ seulement de la dépense nette $\left(l_{t}-r_{t}\right)$ soit financée par l'impôt, tandis que la proportion $\left(1-\alpha_{t}\right)$ est financée par l'emprunt. Nous appellerons $D_{t}$ le coût d'opportunité du capital public financé par l'emprunt (que nous calculerons par la suite). A titre de comparaison, le coût d'opportunité du capital public financé par l'impôt est : (s $\left.v_{t}+1-s_{t}\right)=w_{t}$

La valeur actualisée du projet s'écrit alors :

$$
V A=\sum_{t=0}^{\infty} \frac{\left(b_{t}-c_{t}\right)-\left(e_{t}-r_{t}\right)\left(\alpha_{t} s_{t}\left(v_{t}-1\right)+\left(1-\alpha_{t}\right)\left(D_{t}-1\right)\right)}{(1+a)^{t}}
$$

Une remarque s'impose tout de suite : le coût d'opportunité du capital public n'est pas unique : il y en a autant que de modes de financement, et le coût d'opportunité réel du capital public sera en fait une moyenne pondérée de ces différents coûts. On voit ici la difficulté principale de cette approche (cf aussi la critique du modèle de Marglin par Arrow et Kurz) : le choix d'investissement n'étant pas posé en termes de politique optimale, l'on est obligé de supposer que la répartition du financement entre les différents modes (ici le choix de $\alpha_{t}$ ) est exogène au modèle. Sinon, et si l'on ne fait pas d'hypothèses additionnelles, le projet sera financé à l'époque t à l'aide d'un seul instrument : celui qui rendra le coût d'opportunité du capital public le plus faible.

Supposons néanmoins avec Feldstein que le financement est exogène au choix du projet. La proportion $\alpha_{t}$ est alors fixée. Les deux inconnues du problème sont alors $v_{t}$ et $D_{t}$. Nous présentons leur calcul dans un cas simple

- calcul de v : (Bradford (10)). Supposons qu'une unité d'investissement privé supplémentaire à $t$ se déprécie entièrement à $t+1$, et produise $(1+r p t)$, dont une proportion $s_{t+1}$ est épargnée.

On a alors : $v_{t}=\frac{1+r p t}{1+a}\left(s_{t+1} v_{t+1}+1-s_{t+1}\right)$

si toutes ces grandeurs sont constantes dans le temps, on obtient:

$$
v=\frac{(1-s)(1+r p)}{(1+a)-s(1+r p)}
$$


- calcul de D (à partir de v). On suppose que toutes les grandeurs sont constantes dans le tẹps, et que l'épargne privée est insensible au taux d'intérêt. Cette deuxième hypothèse implique que le coût d'opportunité de la dette est $v$ pour l'année de l'emprunt. La comptabilité des conséquences des intérêts versés (au taux i) est aisée si l'on suppose qu'une proportion 6 de ces intérêts est épargnée, et qu'une fraction $\theta$ de l'intérêt est financée par l'impôt, la fraction (1-O) étant financée par l'emprunt (cette nouvelle rigidité est elle aussi quelque peu induel. Le coût d'opportunité à la date $t$ est alors :

$$
(\theta(s v+1-s)+(1-\theta) D-6 v-(1-6))
$$

On calcule alors aisément :

$$
D=\frac{a v-(1-\theta) i+i(v-1)(\sigma s-b)}{a-(1-\theta) i}
$$

\subsection{Bradford $(1975(10))$.}

Nous avons vu que la théorie de Feldstein suppose que le choix du financement est exogène dans la mesure où l'on a recours aux différents instruments (dette, impôts,...) dans des propositions fixées à l'avance. Par contre, Bradford, de même que Marglin, ne spécifie pas les instruments utilisés pour financer le projet. Il faut de même supposer qu'à ce projet, l'on ne puisse associer qu'une variation et une seule des autres composantes du système. Cette variation, compatible avec les contraintes de l'économie, détermine à son tour les quantités d'investissement privé qui sont "déplacées" par le projet.

Si les hypothèses précédentes sont vérifiées, il suffit comme dans les modèles de Marglin et Feldstein de calculer la somme des deux variations de flux $\left(--, \delta C_{t},--\right)$ et $\left(--, \delta S_{t},--\right)$ évaluée aux deux systèmes de prix fictifs $\left(--, \Pi_{t},--\right)$ et (---, $\Pi_{t} v_{t^{--}}$) où $v_{t}$ est le coût d'opportunité du capital privé, et $\Pi_{t}$ le facteur d'escompte lié à la séquence des taux sociaux de préférence pour le présent.

Supposons maintenant que les capitaux public et privé se déprécient sur une période : à l'époque $t$, on envisage de réaliser un projet public de rentabilité $(1+r g)$. Ce projet sera financé au détriment de la consommation (en proportion $\left(1-\alpha_{t}\right)$ ) et de l'investissement privé (en proportion $\alpha_{t}$ ). Par contre, son produit augmentera.à $(t+1)$ la consommation (en proportion $\left(1-\alpha_{t+1}\right)$ ) et l'investissement privé (en proportion $\alpha_{t+1}$ ). On suppose donc que l'on n'utilisera pas 
le produit pour augmenter l'investissement public à $(t+1) . \underline{\alpha}_{t}$ et $\alpha_{t+1}$ sont supposés définis de façon non équivoque.

L'on peut schématiser ainsi :

\begin{tabular}{|c|c|c|}
\hline & $t$ & $t+1$ \\
\hline$\delta \subset$ & $-\left(1-\alpha_{t}\right)$ & $\left(1+r_{g}\right)\left(1-\alpha_{t+1}\right)$ \\
\hline$\delta s$ & $-\alpha_{t}$ & $\left(1+r_{g}\right) \alpha_{t+1}$ \\
\hline
\end{tabular}

Si $a_{t}$ est le taux social de préférence pour le présent entre $t$ et $(t+1)$, le projet sera adopté si et seulement si :

$$
\frac{1+r}{1+a_{t}} \geq \frac{\left(1-\alpha_{t}\right)+\alpha_{t} v_{t}}{\left(1-\alpha_{t+1}\right)+\alpha_{t+1} v_{t+1}}
$$

Notons les cas importants suivants, en supposant que $v_{t}=v_{t+1}=v$.

i) $\underline{\alpha}_{t} \underline{\alpha}_{t+1}=\underline{b}$ (c'est le cas en particulier quand le financement est réalisé par l'impôt et la propension à épargner le revenu disponible est constante). Le projet est alors adopté si et seulement si : $r_{g} \geq a_{t}$. Le taux d'actualisation est égal au taux social de préférence pour le présent. Nous verrons que c'est le résultat d'Arrow et Kurz en un état stationnaire d'un modèle de croissance (où donc $v_{t}$ est constant ].

ii) $\underline{\alpha}_{t}=1, \alpha_{t+1}=0$ : le critère devient : $(1+r g) \geq v\left(1+a_{t}\right)$. Les répercussions de l'investissement public étant défavorables en termes d'investissement privé, le taux d'actualisation sera, si $v>1$, supérieur au taux social de préférence pour le présent.

iii) $\underline{\alpha}_{t}=0, \alpha_{t+1}=1$ : le critère devient : $(1+r g) \geq \frac{1}{v}\left(1+a_{t}\right)$ (cas inverse de ii)).

iv) l'application de la formule de Bradford (1) à un modèle à deux périodes où les mouvements du système sont univoquement déterminés par la variation de l'investissement public nous permettra de généraliser la formule de Drèze - Sandmo (15) à la réforme (c'est-à-dire à partir d'un état quelconque, et non de l'état optimal) cf. 7.1 .2 . 


\section{- Critiques du modèle de Bradford :}

i) I'on peut formuler la même critique théorique que pour les modèles de Marglin et Feldstein ; en conséquence, la formule (1) ne s'appliquera en réforme que dans un système dont les mouvements sont univoquement définis par ceux de l'investissement public. On pourra aussi l'appliquer en un optimum où par définition tous les coûts d'opportunité du capital public pour les différents degrés de liberté du système sont égaux.

ii) Bradford a lui même critiqué la portée pratique de son modèle (p.897-898). De nombreuses difficultés limitent en effet son emploi :

- Définition du financement : tel hôpital est-il financé par l'impôt sur les sociétés, sur le revenu, par l'emprunt, etc...?

- Même si l'on sait que les autoroutes sont financées par une taxe sur l'essence, rien ne prouve que l'on en ferait pas un autre usage si l'on diminuait le nombre d'autoroutes, ou que l'on augmenterait cette taxe si l'on voulait financer de nouvelles autoroutes. Plus généralement est-il souhaitable de "cloisonner" en affectant certaines recettes à certaines dépenses ?

- L'influence du produit du projet public sur l'investissement privé est-elle aussi bien difficile à déterminer (un parc augmente-t-il la consommation ou l'épargne ?).

Du fait de ces difficultés, Bradford préfère utiliser son résultat pour tester l'égalité du taux d'actualisation déduit de (1) et du taux social de préférence pour le présent (cf quelques exemples numériques p.894). Il en conclut que l'on peut adopter sans trop d'erreur ce dernier taux comme taux d'actualisation.

\subsection{Harberger $(1969$ (21))}

Une approche quelque peu différente de celle de Marglin-Feldstein - Bradford a été proposée par Harberger, puis reprise par Naouri (29). Le raisonnement utilise le concept de surplus dans un modèle à deux périodes. Nous appellerons $i$ le taux d'intérêt du marché financier. En présence d'un taux positif et fixe d'impôt sur les sociétés, le taux réel pour le secteur privé, supposé se financer par actions, est $: i_{p}>i$. De même en présence d'un taux positif et fixe d'impôt sur le revenu, le taux net pour les consommateurs est : $i_{c}<i$. 
Nous traçons ci-dessous les courbes d'offre et de demande sur le marché financier (où $S$ est l'épargne, et I I'investissement) :

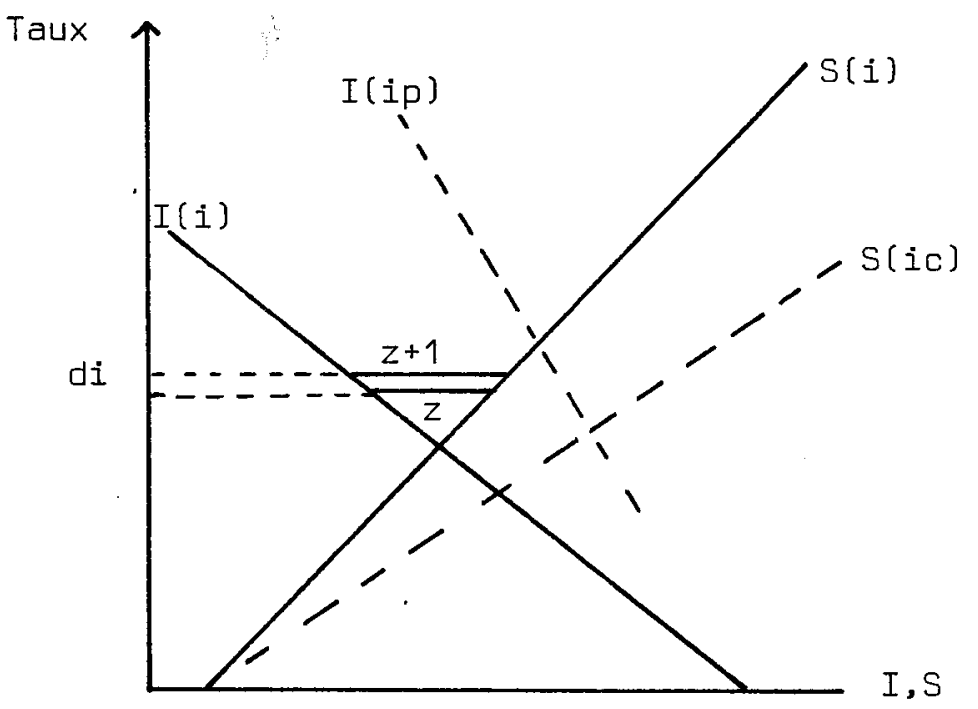

L'investissement public z est supposé être financé par la dette. L'équilibre du màrché financier s'écrit donc : $S(i)=I(i)+z$. Acroissons l'investissement public de 1 . Le taux d'intérêt doit varier de (di) tel que $: I=\left(\frac{\partial S}{\partial i}-\frac{\partial I}{\partial i}\right)$ di

Le taux marginal de substitution entre les deux périodes étant pour les consommateurs $\left(1+i_{C}\right)$, le taux marginal de transformation des producteurs étant (i+ip), le coût d'opportunité du capital public esț :

$$
\frac{\partial S}{\partial i} d i+\left(\frac{1+i p}{1+i c}\right)\left(-\frac{\partial I}{\partial i} d i\right)=\left(1+\frac{\frac{\partial S}{\partial i} i c-\frac{\partial I}{\partial i} i p}{\frac{\partial S}{\partial i}-\frac{\partial I}{\partial i}}\right) \times \frac{1}{1+i_{c}}
$$

Un projet public de rentabilité ( $1+r g$ ) sera donc adopté si et seulement si :

$$
r g \geq \frac{\frac{\partial S}{\partial \dot{i}} i_{C}-\frac{\partial I}{\partial \dot{i}} i p}{\frac{\partial S}{\partial i}-\frac{\partial I}{\partial i}}
$$

(2) (Formule de la moyenne pondérée].

- Critiques du modèle de Harberger :

i) critique méthodologique : la méthode du surplus a pour défaut bien connu d'ignorer les effete revenus. Pour cela, la formule (2) doit être amendée pour en tenir compte; nous montrerons en 7.1.2. que la formule corrigée est : 


$$
r g \geq \frac{\left(\frac{\partial S}{\partial \dot{i}}\right){ }^{u}{ }_{C}-\left(\frac{\partial I}{\partial i}\right) i_{p}}{\left(\frac{\partial S}{\partial i}\right)^{u}-\frac{\partial I}{\partial i}} \quad \text { ["u" signifie : "à utilité constante") }
$$

ii) considérer que la dette est le seul moyen de financer les investissements publics parait fort discutable. Il n'est d'ailleurs pas sûr que face à un marché financier comprenant des distorsions, la politique optimale de l'Etat soit d'utiliser cet instrument dans le sens de l'emprunt et non du prêt (cf. (35) essai III).

- Par cet article, Harberger a amendé sa position théorique d'avant 1968 en reconnaissant que la dette publique peut augmenter le taux d'intérêt, et donc accroître l'épargne disponible. Il estime cependant qu'en pratique la faible sensibilité de l'épargne au taux d'intérêt égalise le taux d'actualisation et le taux d'intérêt auquel sont confrontés les producteurs privés (supposé égal à la productivité marginale du capital privé en l'absence de primes de risque).

Notons enfin que Baumol dans son article précurseur de 1968 (6), où il pose le problème du taux d'actualisation en termes d'optimum second, propose aussi une formule de type (2) ou de moyenne pondérée (bien qu'il adopte une position ambigue en donnant aussi des arguments en faveur de la productivité marginale du capital privé).

7. Modèles de politique optimale.

Nous distinguerons deux types d'approche selon que l'auteur considère une génération unique prenant ses décisions sur un nombre fini de marchés à terme, ou bien un modèle de croissance, dont il fait l'étude des états stationnaires. Cette dernière approche se situe dans la lignée d'Arrow-Kurz. Dans la première catégorie peuvent être placés les modèles de prix publics optimaux, qui, réinterprétés en termes intertemporaux ont pour sous-produit un système de taux d'actualisation (par exemple Diamond-Mirrlees(13)). Un modèle plus directement conçu pour l'étude du taux d'actualisation est celui de Sandmo et Drèze (15); nous montrerons que leur résultat principal est valable non seulement en un optimum, mais aussi en réforme (à partir d'un état initial quelconque). Enfin nous évoquerons les idées de Thédié (34) sur le taux d'actualisation en présence de contraintes de financement, et nous en donnerons une formulation précise à l'aide du modèle de Boiteux ( 8 ). 


\subsection{Modèles comportant des marchés à terme}

7.1.1 Taxation aptimale (Diamond-Mirrlees (1971 (13)), Diamond (1973 (14))

L'hypothèse principale du modèle de Diamond-Mirrlees, et de sa traduction en termes intertemporels (Diamond) est la possibilité de déconnecter complètement les sphères de la production et de la consommation : d'abord par une taxation optimale des biens, ensuite par la non-transmission des profits des entreprises privées aux consommateurs (soit grâce à la confiscation des profits, soit en supposant des rendements d'échelle constants). Il est alors intuitif que la sphère de la production doit être globalement efficace, bien que l'optimum ne soit pas premier ; pour notre objet, les taux marginaux de productivité doivent être égaux dans les secteurs public et privé ${ }^{(1)}$.

\subsubsection{Financement_par_l'emprunt_en_présence_d'un_impôt_sur_le _bénéfice des socoiétés (Drèze - Sandmo (1971 (15)).}

Drèze et Sandmo ont proposé un modèle à deux périodes où la distorsion du marché financier prend la forme d'un taux fixe t d'impôt sur le bénéfice des sociétés. Le financement de l'investissement public z en première période se fait par l'emprunt. L'épargne privée se partage donc entre investissement public $z$ et investissement privé $y$. Si $\omega$ est la dotation initiale de l'économie, et si $f$ et $g$ sont les fonctions de production, supposées croissantes, strictement concaves et différentiables, des secteurs privé et public, les contraintes physiques de l'économie s'écrivent pour les deux périodes ( $\mathrm{sic} \mathrm{c}_{1}$ et $\mathrm{c}_{2}$ sont les consommations à $t=1,2$ ) :

$$
\left\{\begin{array}{l}
c_{1}+y+z=\omega \\
c_{2}=f(y)+g(z)
\end{array}\right.
$$

i) comportement du consommateur : celui-ci maximise son utilité $u\left(c_{1}, c_{2}\right)$ supposée strictement concave sous sa contrainte budgétaire $:\left(c_{1}+\frac{C_{2}}{1+i} \leq R\right)$

(1) Le modèle utilisé par Diamond pour en donner la traduction intertemporelle est un modèle à générations imbriquées. L'efficacité de la production est de même désirable pour une taxation optimale, mais ne l'est généralement pas en cas de taxation partielle. 
où $R$ est son revenu intertemporel et i le taux d'intérêt du marché financier.

L'on appelle $v(i, R)$ la fonction d'utilité indirecte, et l'on a les égalités classiques :

- Siutsky $-\frac{\partial c_{1}}{\partial i}=\left(\frac{\partial c_{1}}{\partial i}\right)^{U}+\frac{c_{2}}{(1+i)^{2}} \frac{\partial c_{1}}{\partial R}$

$$
\left\{\begin{array}{l}
\frac{\partial c_{1}}{\partial R}+\frac{1}{1+i} \frac{\partial c_{2}}{\partial R}=1 \\
\frac{\partial c_{1}}{\partial i}+\frac{1}{(1+i)} \frac{\partial c_{2}}{\partial i}=\frac{1}{(1+i)^{2}} c_{2}
\end{array}\right.
$$

- Roy $\quad \frac{\partial v}{\partial i}=\frac{\partial v}{\partial R} \times \frac{C_{2}}{(1+i)^{2}}$

Pour une petite variation $(d i, d R)$ du taux d'intérêt et du revenu, la variation de l'utilité indirecte est :

$$
d v=\frac{\partial v}{\partial R} d R+\frac{\partial v}{\partial i} d i=\frac{\partial v}{\partial R}\left(\frac{c_{2}}{(1+i)^{2}} d i+d R\right) \sim \frac{c_{2}}{(1+i)^{2}} d i+d R
$$

ii) comportement du producteur : celui-ci maximise son profit intertemporel :

$$
\operatorname{Max}\left(-y+\frac{f(y)}{1+\frac{i}{1-t}}\right)
$$

La productivité marginale du capital privé est donc égale au taux d'intérêt auquel est confronté le producteur : $f^{\prime}(y) \equiv 1+\mathrm{rp}=1+\frac{i}{1-t}$ L'impôt sur les sociétés introduit donc un biais entre taux de préférence pour le présent, égal au taux d'intérêt, et le taux de productivité marginale de l'investissement privé.

iii) une variation du système est entièrement décrite par (dz,di,dR). Cette variation doit vérifier les deux équations de contraintes physiques :

$$
\begin{aligned}
& d z=\left(-\frac{\partial c_{1}}{\partial i}-\frac{\partial y}{\partial i}\right) d i-\frac{\partial c_{1}}{\partial R} d R \\
& g^{\prime}(z) d z=\left(\frac{\partial c_{2}}{\partial i}-f^{\prime}(y) \frac{\partial y}{\partial i}\right) d i+\frac{\partial c_{2}}{\partial R} d R
\end{aligned}
$$

Dès lors il est possible d'exprimer les variations des instruments ( $d i, d R$ ) en fonction uniquement de la variation de l'investissement public dz (remarquons que nous sommes dans le cas d'univocité qui est l'hypothèse implicite aux modèles de réforme décrits dans la première partie). 
On obtient :

$$
\begin{aligned}
& d i=\frac{d z}{\Delta}\left[\frac{\partial c_{2}}{\partial R}+g^{\prime}(z) \frac{\partial c_{1}}{\partial R}\right] \\
& d R=\frac{d z}{\Delta}\left[-\frac{\partial c_{1}}{\partial \dot{i}} g^{\prime}(z)-\frac{\partial y}{\partial \dot{i}} g^{\prime}(z)-\frac{\partial c_{2}}{\partial \dot{i}}+f^{\prime}(y) \frac{\partial y}{\partial \dot{i}}\right]
\end{aligned}
$$

avec :

$$
\Delta=(1+i)\left[-\left(\frac{\partial c}{\partial i}\right)^{u}\right]+\left[(1+i)+\frac{\partial c}{\partial R}\left(f^{\prime}(y)-1-i\right)\right]\left(-\frac{\partial y}{\partial i}\right)
$$

Remarque : Si le taux d'impôt sur les bénéfices des sociétés t est positif $\left[f^{\prime}(y) \equiv 1+r p\right.$ ést supérieur à $\left.(1+i)\right]$, et si le bien de consommation de première période est supérieur, $\Delta$ est positif. Dans le cas général, nous serons amenés à supposer : $\Delta>0$, ce qui semble être une hypothèse raisonnable *.

$$
\text { On peut alors substituer (5) et (6) dans (4); I'on trouve }
$$

$$
d v \sim\left[g^{\prime}(z)\left(-\left(\frac{\partial c_{1}}{\partial i}\right)^{u}-\frac{\partial y}{\partial \dot{i}}\right)-(1+i)\left(-\frac{\partial c_{1}}{\partial \dot{i}}\right)^{u}-(1+r p)\left(-\frac{\partial y}{\partial i}\right)\right] \frac{d z}{\Delta}
$$

Proposition 1 Si l'on suppose que $\Delta$ est positif, en un état initial (o) quelconque, le taux d'actualisation ag pour les investissements publics est donné pour la "formule de la moyenne pondérée" (des taux de préférence pour le présent et de productivité marginale du capital privée par la consommation et l'investissement "déplacés") :

$$
a g(a)=\left[\frac{i\left(-\frac{\partial c_{1}}{\partial i}\right)^{u}+r_{p}\left(-\frac{\partial y}{\partial \dot{j}}\right)}{\left(-\frac{\partial c_{1}}{\partial i}\right)^{u}+\left(-\frac{\partial y}{\partial \dot{i}}\right)}\right] \text { (o) }
$$

\footnotetext{
Le cas $\Delta<0$ est quelque peu pathologique : il faut alors diminuer l'investissement public si la productivité marginale du secteur public est supérieure au taux donné par la formule de la moyenne pondérée. Grosso modo, ce cas peut se présenter en cas de très importantes subventions $\left(f^{\prime}(y)<1+i\right)$; dans ce cas, un projet public très rentable ne fait qu'accroître la distorsion. Ce fait n'est pas sans rappeler le résultat selon lequel, dans le modèle de Diamond-Mirrlees et en réforme, le système de prix publics fictifs est l'opposé du système de prix à la production quand des inefficacités temporaires dans le secteur privé sont désirables (cf. [19]).
} 
Rappelons que Sandmo et Drèze n'ont montré la formule (7) qu'en un état optimal. Nous avons classé ce modèle avec les modèles de politique optimale à cause de son origine.

Remarque : L'on retrouve ici, en tenant compte de l'effet revenu, la formule de Harberger (démontrée en 6.4).

iv) Proposition 2 La formule (1), ou formule de Bradford, permet de retrouver la proposition 1.

Nous avons remarqué qu'à une variation dz de l'investissement public correspondait une variation et une seule des instruments : (di,dR) satisfaisant les contraintes de l'économie (en d'autres termes il n'existe pas d'autre degré de liberté que l'investissement public). Nous sommes donc en mesure d'appliquer la formule de Bradford (cf. 6.3).

Le respect des préférences individuelles dans le madèle de DrèzeSandmo impose i comme taux social de préférence pour le présent.

Comme il n'y a pas de réinvestissement en deuxième période

$$
\left\{\begin{array}{l}
\alpha_{t+1}=0 \\
v_{t+1}=0
\end{array}\right.
$$

$\alpha_{t}$ est défini par : $\alpha_{t} \equiv-\frac{\partial y}{\partial i} d i$, où di est la variation du taux d'intérêt associéè̀ $d z=+1$.

Le coût d'opportunité du capital privé est, en l'absence de réinvestissement : $\quad v=\frac{1+\Gamma P}{1+i}$.

La formule (1) s'écrit alors: $\frac{g^{\prime}(z)}{1+i} \geq\left(1-\alpha_{t}\right)+\alpha_{t}\left(\frac{1+r p}{1+i}\right)$.

Il suffit alors de calculer $\alpha_{t}$, di étant donné par (5). On peut alors écrire (en supposant $\Delta>0$ ) :

$$
g^{\prime}(z) \geq(1+i)\left(\frac{-\left(\frac{\partial c_{1}}{\partial i}\right)^{u}}{-\left(\frac{\partial c}{\partial i}\right)^{u}-\frac{\partial y}{\partial i}}\right)+(1+r p)\left(\frac{-\frac{\partial y}{\partial i}}{-\left(\frac{\partial c_{1}}{\partial i}\right)^{u}-\frac{\partial y}{\partial i}}\right)
$$

ce qui est bien la formule de la proposition 1. 
Notons enfin que Diamond (12) avait démontré une formule analogue à (7), mais seulement à l'optimum, de même que Drèze-Sandmo.

\subsubsection{Coût_de_rareté des_fonds_publics (Thédié 1974 (34))}

i) Dans un article intitulé "A challenge to the monopoly of the rate of discount in investment choices", Thédié propose d'introduire un cout de rareté des fonds publics lorsque le secteur public fait face à des contraintes de financement. supposons pour simplifier que le cout à $t: c_{t}^{P} d^{\prime} u n$ projet $p(p=1,--P)$ soit uniquement monétaire, tandis que le bénéfice à $t: b_{t}^{P}$ est monétaire ou non.

L'idée de Thédié est la suivante : Dans un premier temps, le décideur résoud un programme ne comportant pas les contraintes de financement. Il en résulte alors un système de taux d'escompte ${ }_{t}$ qui du fiait de L'optimalité du programme, sont interprétés en termes de valeur d’échange : $\pi_{t}^{\prime}$ unités de consommations à $t$ peuvent être échangées indifféremment contre $\pi_{t}$ unités de consommation à t'. Le critère pour le choix du projet est alors :

$$
\sum_{t} \pi_{t}\left(b_{t}^{P}-c_{t}^{P}\right) \geq 0
$$

Dans un deuxième temps, I'on introduit une contrainte de financement à chaque période $t$ sous la forme d'un plafond $J_{t}$. On suppose que le secteur public est suffisamment petit pour que l'influence de ces contraintes ne modifie que peu la séquence des $\pi_{t}$. Le nouveau programme (pour le secteur public] s'écrit :

$$
\left\{\begin{array}{l}
\operatorname{Max} \sum_{t} \pi_{t}\left(\sum_{P}\left(b_{t}^{P}-c_{t}^{P}\right) x^{P}\right) \\
\sum_{P} c_{t}^{P} x^{P} \leq J_{t} \quad\left(B_{t}\right) \\
x^{P}=0 \text { ou } 1 .
\end{array}\right.
$$

où $x^{P}$ est une variable de décision. Il est plus simple de supposer que les projets sont divisibles, et de remplacer la contrainte de décision par la double contrainte : $\begin{cases}x^{P} \geq 0 & \left(\eta^{P}\right) \\ x^{P} \leq 1 & \left(\psi^{P}\right)\end{cases}$ 
Il est alors aisé de montrer alors qu'un projet retenu (ce qui implique $\eta^{P}=0$ ) est caractérisé par :

$$
\sum_{t} \pi_{t}\left(t_{t}^{P}-\left(1+\frac{B_{t}}{\pi_{t}}\right) c_{t}^{P}\right) \geq 0
$$
On appelle alors $\left(1+\frac{\beta_{t}}{\pi_{t}}\right)$ le coefficient de rareté des fonds publics à
l'époque $t$.

ii) Nous allons reformuler l'idée de Thédié dans un cadre plus général, où nous n'aurons pas besoin de procéder à l'optimisation à deux niveaux comme le fait Thédié.

Le cadre de notre modèle sera donné par l'article bien connu de Boiteux ( 8 ). Nous supposerons qu'il y a $T$ périodes, et $H$ biens physiques (et donc TH biens économiques). L'économie comprend m consommateurs $\{i=1, \cdots, m)$ et $n$ entreprises $(j=1,--n\}$. Le système de prix unique est appelé $p=\left\{--, p_{h t},--\right\}$.

Les transferts forfaitaires sont possibles, et le revenu intertemporel du consommateur $i$ est appelé Ri . De façon classique, le consommateur a une fonction d'utilité indirecte $v_{i}(p$, Ri) et une fonction de demande $x^{i}(p$, Ri) supposée différentiable.

De même I'entreprise privée $j$ a une fonction d'offre $y^{j}(P)$ supposée différentiable.

Le secteur public fait face à une contrainte de financement à chaque période $t$ : la valeur actualisée des inputs ne doit pas dépasser $J_{t}$. Nous décrivons de deux manières la technologie du secteur public :

a) approche "choix des projets" : Le gouvernement a recencé P projets incompatibles $(p=1,--, P)$ caractérisés chacun par des échéanciers d'inputs $\left\{a_{h t}^{P}\right\}$ et d'outputs $\left\{b_{h t}^{P}\right\}$, et l'on supposera désormais que tous les avantages et coûts sont marchands. Les projets seront supposés divisibles afin de remplacer la contrainte de décision par une double contrainte linéaire $\left\{\begin{array}{l}x^{P} \geq 0 \\ x^{P} \leq 1\end{array} \quad\right.$ (en sachant bien que $x^{P} \quad$ (sauf peut être pour le projet marginal) sera égal à l'une des deux valeurs extrêmes). 
La contrainte de financement à $t$ s'écrit alors :

$$
\sum_{p=1}^{P} p_{h t} a_{h t}^{P} x^{P} \leq J t
$$

Nous allons maintenant caractériser un optimum de Pareto de cette économie : pour cela, nous résolvons le programme (I) :

$$
\text { (I) } \begin{cases}\operatorname{Max}\left[\lambda_{1} v_{1}\left(p, R_{1}\right)\right] & \left(\lambda_{1}>0\right) \\ v_{i}(P, R i) \geq v^{2} \quad(\lambda i) \quad i=2,-\cdots, m \\ \sum_{i} x_{h t}^{i}(p, R i) \leq \sum_{j} y_{h t}^{j}(P)+\sum_{P}\left(b_{h t}^{P}-a_{h t}^{P}\right) x^{P} \quad\left(\mu_{h t}\right) \\ \sum_{p} \sum_{h} P_{h t} a_{h t}^{P} x^{P} \leq J_{t} \\ x^{P} \leq 1 & (\beta t) \\ x^{P} \geq 0 & \left(\psi^{P}\right)\end{cases}
$$

Nous appellerons : (h't', ht) $=\sum_{j} \frac{\partial y_{h^{\prime} t^{\prime}}^{j}}{\partial P_{h t}}-\sum_{i}\left(\frac{\partial x_{h^{\prime} t^{\prime}}^{i}}{\partial P_{h t}}\right) u_{i}=$ cste

(c'est-à-dire la variation d'offre nette en ( $h$ ', $t^{\prime}$ ) quand le prix de (h, $t$ ) varie, les demandes étant compensées]. L'écriture des conditions du premier ordre donne :

$$
\sum_{h \cdot t}, \mu_{h \prime t},(h \cdot t, h t)+\beta_{t}\left(\sum_{p} a_{h t}^{P} x^{P}\right)=0
$$

qui sont les relations de Boiteux pour $\left(\beta_{t}\right)$ ce modèle (pour leur interprétation, voir Boiteux (8). Rappelons que $\left.: \sum_{h^{\prime} t}, P_{h^{\prime} t},\left(h^{\prime} t^{\prime}, h_{t}\right)=0\right)$.

La dérivation par rapport à $x^{P}$ donne :

$$
\sum_{t} \sum_{h} \mu_{h t}\left[b_{h t}^{P}-a_{h t}^{P}\right]-\sum_{t}\left(\sum_{h} p_{h t} a_{h t}^{P}\right) \beta_{t}-\psi^{P}+n^{P}=0
$$

Un projet adopté p sera tel que $\eta^{P}=0$. Puisque $\psi^{P}$ est positif ou nul :

$$
\sum_{t}\left(\sum_{h} \mu_{h t} b_{h t}^{P}-\sum_{h} \mu_{h t}\left(1+\frac{\beta_{t} P_{h t}}{\mu_{h t}}\right) a_{h t}^{P}\right) \geq 0
$$

Si on choisit le bien $H$ comme numéraire, le système des prix relatifs fictifs associé est : $\hat{\mu}$ tel que : $\hat{\mu}_{h t}=\frac{\mu_{h t}}{\mu_{H t}}$. Un projet adopté p est caractérisé par : 


$$
\sum_{t}\left[\frac{\mu_{H t}}{\mu_{H 1}}\right]\left[\sum_{h} \hat{\mu}_{h t} b_{h t}^{P}-\sum_{h} \hat{\mu}_{h t}\left(1+\beta_{t} \frac{P_{h t}}{\mu_{h t}} a_{h t}^{P}\right] \geq 0\right.
$$

La formule (10) a bien la forme préconisée par Thédié avec :

$$
\begin{aligned}
& \frac{\mu_{H t}}{\mu_{H 1}} \text { est le facteur d'escompte à } t \text { lié aux valeurs sociales de } H . \\
& \dot{\mu}_{h t} \text { est le prix relatif fictif du bien h à } t \text {. } \\
& \left(1+\beta_{t} \frac{P_{h t}}{\mu_{h t}}\right) \text { est le coût de rareté associé au bien } h \text { à } t .
\end{aligned}
$$

Si la contrainte de financement à l'époque $t$ n'est pas saturée, ce coût de rareté est égal ̀̀ 1.

\section{b) Fonctions de production par périodes}

On supposera désormais que la technologie du secteur public est décrite non plus par une liste de projets incompatibles, mais par une fanction de production propre à chaque période (pour plus de détails, cf. Malinvaud (26)) : le vecteur $a_{t}$ des inputs à $t$ et le vecteur $b_{t+1}$ des outputs à $t+1$ doivent vérifier :

$$
g_{t}\left(a_{t}, b_{t+1}\right) \leq 0 \quad\left(\Phi_{t}\right)
$$

L'on supposera que $g_{t}$ est différentiable.

La contrainte de financement à $t$ s'écrit :

$$
\sum_{h} P_{h t} a_{h t} \leq J_{t} \quad\left(\beta_{t}\right)
$$

et la contrainte de rareté du bien h à l'époque t est :

$$
\sum_{i} x_{h t}^{i}(p, R i) \leq \sum_{j} y_{h t}^{j}(p)+b_{h t}-a_{h t} \quad\left(\mu_{h t}\right)
$$

On obtient alors le programme (II) de caractérisation d'un optimum de Pareto d'une telle économie : 
Les relations de Boiteux s'écrivent ici :

$$
\left\{\begin{array}{l}
\operatorname{Max}[\lambda 1 \vee v 1(P, R 1)] \\
v i(P, R i) \geq \overline{v i} \quad(\lambda 1>0) \\
\sum_{i} x_{h t}^{i}(P, R i) \leq \sum_{j} y_{h t}^{j}(P)+b_{h t}-a_{h t} \quad\left(\mu_{h t}\right) \\
\sum_{h} P_{h t} a_{h t} \leq J_{t} \\
g_{t}\left(a_{t}, b_{t+1}\right) \leq 0 \quad\left(\beta_{t}\right)
\end{array}\right.
$$$$
\left.\sum_{h \cdot t} \cdot \mu_{h \prime t},(h \cdot t), h t\right)+\beta_{t} a_{h t}=0
$$

Les dérivations du Lagrangien par rapport à $a_{h t}$ et à $b_{k t+1}$ donnent les expressions :

$$
\begin{gathered}
-\mu_{h t}-\beta_{t} P_{h t}-\Phi_{t} \frac{\partial g_{t}}{\partial a_{h t}}=0 \\
\mu_{k t+1}-\Phi_{t} \frac{\partial g_{t}}{\partial b_{k t+1}}=0
\end{gathered}
$$

On peut voir que les conditions d'efficacité intertemporelle de Malinvaud (26) ne sont plus vérifiées, du fait des contraintes de financement :

$$
\frac{\partial g_{t-1} / \partial b_{k t}}{\partial g_{t-1} / \partial b_{h t}} \text { et } \frac{\partial g_{t} / \partial a_{k t}}{\partial g_{t} / \partial a_{h t}} \text { ne sont en général pas égaux. }
$$

Considérons maintenaht une petite variation des inputs $\delta a_{t}$ et des outputs $\delta b_{t+1}$ vérifiant :

$$
\sum_{h} \frac{\partial g_{t}}{\partial a_{h t}} \delta a_{h t}+\sum_{k} \frac{\partial g_{t}}{\partial b_{k t+1}} \delta b_{k t+1}=0
$$

Les équations (12), (13) et (14) permettent de déduire :

$$
\sum_{k} \mu_{k t+1} \delta b_{k t+1}-\sum_{h} \mu_{h t}\left(1+\beta_{t} \frac{P_{h t}}{\mu_{h t}}\right) \delta a_{h t}=0
$$

(15) est l'équivalent sur les deux périodes successives $t$ et $t+1$ de la formule (10). Les $\left\{\mu_{h t}\right\}$ sont les valeurs sociales attachées aux biens économiques. 
On retrouve d'autre part la même expression qu'en (10) pour le coût de rareté des fonds publics attaché au bien h à $t:\left(1+\beta_{t} \frac{P_{h t}}{\mu_{h t}}\right)$.

Une autre interprétation de la formule (15) est que pour une entreprise publique, $\mu_{h t}$ est le prix fictif de vente de $h$ à $t$, tandis que $\left(\mu_{h t}+\beta_{t} P_{h t}\right)$ est le prix fictif d'achat de h à t. Les contraintes sur le financement des inputs introduisent une distorsion entre les deux systèmes de prix d'achat et de prix de vente dans un contexte de décentralisation.

Nous avons donc formalisé les idées de Thédié sur le coût de rareté des fonds publics. Ce type de modèle est cependant critiquable dans la mesure où il est difficile de justifier théoriquement la présence d'une contrainte de financement rigide. De telles contraintes peuvent cependant exister dans la pratique ${ }^{(*)}$.

\subsection{Modèles de croissance et étude des états stationnaires}

A la suite du développement de la théorie de la croissance dans les années soixante, Arrow (2), puis Arrow et Kurz (5) ont eu l'idée d'appliquer celle-ci au problème de choix d'investissements publics en situation d'optimum second. Pour corriger les distorsions existant dans l'économie, Arrow et Kurz se donnent un ou plusieurs instruments, et montrent dans la tradition de Tinbergen qu'avec suffisamment d'instruments, il est possible d'atteindre un optimum premier (mais le simple décompte des instruments ne suffit évidemment pas pour obteniri un tel résultat). Kay (24) a approfondi leur analyse, tandis que Pestiau et Possen (31) l'ont appliquée à un modèle à deux classes. Pestiau (30) a quant à lui considéré un modèle de croissance à générations imbriquées. Il ne peut être question de décrire ici tous ces modèles, et nous n'en donnerons que les grandes lignes.

\footnotetext{
* Voir la notion de "coefficient de restriction des crédits" dans la circulaire des routes.
} 
a) Arrow (1966 (2)) et Arrow-Kurz (1970 (5))

Nous avons déjà noté que le fait que le taux de rentabilité de l'investissement public soit inférieur à celui de l'investissement privé n'implique pas qu'il y ait sur-investissement public. La solution d'optimum second peut exiger un taux plus faible quand il y a des contraintes sur le champ de l'investissement public ou quand le gouvernement est restreint dans son choix.

B) La règle d'Arrow (2) énonce que la politique optimale de la Puissance Publique en un état stationnaire est en général d'investir jusqu'à ce que la productivité marginale du capital public soit égale au taux social de préférence pour le présent (alors égal au taux pur de préférence pour le présent), et ceci quelque soit la productivité marginale du capital privé. Quel est son champ d'application ? Rappelons que Bradford (cf. section 6) a montré que cette conclusion dépend de l'hypothèse selon laquelle la diminution $\alpha_{t}$ de la formation de capital privé due à une unité supplémentaire d'investissement public à $t$, est égale à l'augmentation $\alpha_{t+1}$ de la formation de capital privé causée par une unité supplémentaire de bénéfices à $(t+1)$; en d'autres termes, les coûts sociaux d'opportunité des coûts et bénéfices sont égaux (puisqu'en un état stationnaire : $v_{t}=v_{t+1}$ ), et le taux sacial de préférence pour le présent est alors le seul élément pertinent au choix des investissements publics.

Typiquement, si la Puissance Publique est à même de contrôler le ou les revenus des consommateurs-épargnants (mais pas nécessairement par des transferts forfaitaires), et si les bénéfices et coûts des investissements publics sont purement monétaires, la règle d'Arrow s'applique (*). C'est dans cette optique qu'elle a été démontrée pour des propensions à épargner rigides (Arrow (2), Arrow-Kurz (5), ch. VI), et généralisée à des comportements d'épargne plus classiques (Arrow-Kurz (5), ch. VIII,

\footnotetext{
* En un optimum, les coûts d'opportunité correspondant aux différents degrés de liberté du système sont égaux. En particulier, si la Puissance Publique peut agir sur les revenus (que les instruments correspondants distordent ou nonj., les coûts d'opportunité des coûts et bénéfices d'un investissement public sont égaux en un état stationnaire (chaque franc soustrait au revenu disponible diminue l'épargne privée d'une quantité égale à l'accroissement d'épargne privée due à une augmentation unitaire du revenu disponible).
} 
Pestiau (30)), ainsi qu'à une économie à plusieurs classes (Pestiau-Possen (31), et (35) essai III).

Nous évoquons maintenant le modèle de croissance utilisé par Arrow et 'Kurz :

- Critère : Comme dans la plupart des modèles de croissance est introduit, pour des raisons de convergence, un taux pur $\rho$ de préférence pour le présent. De sarte que le fondement de la préférence pour le présent de la collectivité est le double jeu de la décroissance de l'utilité marginale et du taux pur (voir notre discussion en 1). Si l'accord paraît complet pour écrire l'utilité comme fonction de la consommation par tête $c_{t}$, tous les auteurs n'ont pas la même position sur la forme finale du critère en cas de croissance démographique. L'argument d'Arrow et Kurz est le suivant : considérons deux Iles de populations $N_{1}$ et $N_{2}$. Soit $C$ une quantité à distribuer entre les deux populations $\left(C=C_{1}+C_{2}\right.$ ). Sous l'hypothèse d'identité des individus, et la condition qu'une fonction de bien-être social doit dans ce cas aboutir à une répartition égalitaire, la fonction de bien-être collectif doit être :

$$
W=N_{1} U\left(\frac{C_{1}}{N_{1}}\right)+N_{2} U\left(\frac{C_{2}}{N_{2}}\right)
$$

Dans notre cadre intertemporel, la fonction de bien-être social sera, si la population à l'époque $t$ est $: e^{n t}$,

$$
W=\int_{0}^{\infty} e^{-\rho t}\left[e^{+n t} U\left(c_{t} e^{-n t}\right)\right] d t=\int_{0}^{\infty} e^{-(\rho-n) t} U\left(c_{t}\right) d t \text { où } c_{t} \equiv \frac{c_{t}}{e^{n t}} \text {. }
$$

On supposera pour assurer la convergence de cette intégrale que :

$$
\rho>n
$$

- L'étude d'Arrow et Kurz est axée sur la "contrôlabilité" du système :

Définition ( $p$ 120) Une politique est dite contrôlable à partir d'un ensemble donné d'instruments s'il existe des valeurs de ces instruments, variant en général au cours du temps, qui font suivre cette politique aux secteurs public et privé. 
La politique à laquelle Arrow et Kurz pensent est bien sûr la politique d'optimum premier. On peut aisément montrer (voir par exemple ArrowKurz ch. IV) que cette dernière exige que les productivités marginales du capital ṕrivé et du capital public soient à chaque instant égales, et, en un état stationnaire, égales au taux pur de l'économie (ou encore au taux social de préférence sur le présent puisque l'utilité marginale de la consommation est alors constante).

La règle d'Arrow est que même si la politique d'optimum premier n'est pas contrôlable à partir des instruments (d'où la recherche d'une politique d'optimum second), la productivité marginale du capital public doit, en général, être égale en un état stationnaire au taux social de préférence pour le présent.

- Donnons une preuve heuristique de la règle d'Arrow en raisonnant en termes de variations autour d'un optimum; pour cela supposons que (pour simplifier l'écriture), la population soit constante, et que le gouvernement dispose d'instruments fiscaux grâce auxquels il augmente l'investissement public à la date o d'1F. On suppose qu'il règle par la suite ses instruments fiscaux de manière à ne pas modifier ses revenus à $t$ par rapport à l'esquisse initiale $(\forall t \geq 1)$. L'investissement public supplémentaire à $t=0$ est supposé avoir une rentabilité $(1+r g)$ à $t=1$, et nulle après. Comme il a été financé à partir du revenu disponible des consommateurs qui ont un taux d'épargne s à partir de ce revenu disponible, et en supposant que le capital privé a une productivité marginale $(1+\mathrm{rp})$ l'année postérieure à l'investissement, nulle après, l'investissement public supplémentaire entraîne :

- à $t=0$ une diminution de consommation égale à $(1-s)$

- à $t=1$ une augmentation de revenu disponible égale à $(1+r g)-s(1+r p)$ dont une partie s est épargnée et une partie (1 - s) est consommée.

- à $t=2$ une augmentation du revenu disponible des consommateurs de $(1+r p) s[(1+r g)-s(1+r p)]$ dont une proportion s est épargnée et ainsi de suite... 
Le supplément initial d'investissement public entraîne donc une variation du flux de consommation ayant pour valeur actualisée :

$$
\begin{aligned}
\sum_{t=0}^{\infty} \frac{\delta C_{t}}{(1+\rho)^{t}} & =(1-s)\left[-1+\sum_{t=1}^{\infty} \frac{\left[(1+r p) s{ }^{t-1}[(1+r g)-s(1+r p)]\right.}{(1+\rho)^{t}}\right] \\
& =(1-s) \frac{r g-p}{(1+\rho)-s(1+r p)} \text { si } 1+\rho>(1+r p) s
\end{aligned}
$$

On voit alors qu'il y a deux possibilités :

1) $1+p>(1+r p) s \quad$ Dans ce cas, le taux d'actualisation ag est tel que ni un investissement initial, ni un désinvestissement initial ne peuvent améliorer la fonction objective et donc : $\underline{a g}=\rho$ c'est le résultat d'Arrow.

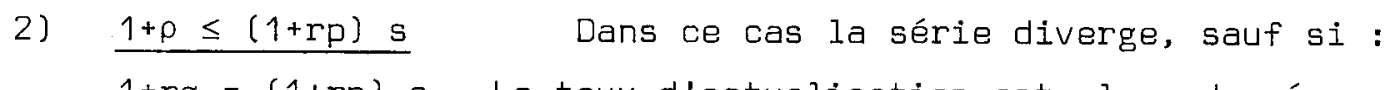
$1+r g=(1+r p) s$. Le taux d'actualisation est alors donné par :

$$
\underline{1+a g}=(1+r p) s
$$

Ce résultat est dû à Kay (24), qui reprend le modèle de croissance utilisé par Arrow et Kurz. Le taux d'actualisation est donc :

$$
a g=\max [\rho, s(1+r p)-1]
$$

ii) Pestiau (1974 (30́))

Pestiau utilise le modèle classique de croissance à générations imbriquées (chaque génération vivant deux périodes). Il est alors plus aisé que dans le modèle d'Arrow et Kurz de manier des comportements d'épargne non rigides. Les investissements publics sont financés à partir de deux instruments fiscaux (impôts sur le salaire et sur l'intérêt) et éventuellement de la dette. Les deux instruments fiscaux ont des effets nocifs sur l'efficacité de l'économie : ils tendent à diminuer respectivement l'offre de travail et 1 'épargne. 
Les résultats de Pestiau sont pourtant dans la lignée de ceux d'Arrow-Kurz :

- si le gouvernement dispose des deux instruments fiscaux seulement, la productivité marginale du capital public rg sera, en un état stationnaire, égale au taux social de préférence pour le présent.

Par contre, la productivité marginale rp du capital privé sera affectée par les distorsions : on trouve en effet :

$$
1+r g=\frac{(1+r p)\left(1-\varepsilon_{i}\right)+(1+i) \varepsilon_{i}}{1-\frac{\theta w}{w} \varepsilon w}
$$

où $i$ est le taux d'intérêt, $\varepsilon_{i}$ l'élasticité compensée de la consommation de deuxième période par rapport à $(1+i)$, EW l'élasticité compensée de l'offre de travail par rapport au taux de salaire $w$ et $\frac{\theta w}{w}$ le taux d'imposition sur le travail.

- Si le gouvernement dispose des trois instruments (deux instruments fiscaux et la dette), les productivités marginales du capital public et privé sont égales entre elles et égales au taux social de préférence pour le présent.

\section{r) Pestiau-Possen (1977) (31)).}

Pestiau et Possen divisent l'économie en deux classes, et supposent que le gouvernement, sur la base d'une fonction d'utilité collective, désire réaliser une certaine redistribution entre ces classes. Si le nombre des instruments est faible, le gouvernement doit rechercher un équilibre entre justice et efficacité, du fait qu'une redistribution des "capitalistes" vers les "travailleurs" diminue l'épargne privée.

Par rapport à Arrow-Kurz et à Pestiau, les causes de distorsions sont donc différentes. Cependant les résultats présentent une certaine conformité par rapport aux précédents :

- en un état stationnaire, le taux d'actualisation est égal au taux social de préférence pour le présent; 
- si le nombre d'instruments est trop faible, ou si ceux-ci sont inadéquats, la productivité marginale du secteur privé diffère du taux social de préférence pour le présent

\section{Conclusion}

Nous avons vu que le résultat d'Arrow (égalité du taux d'actualisation et du taux social de préférence pour le présent en croissance équilibrée) est relativement robuste; la remarque de Bradford donne la condition de sa validité. D'autre part le mérite d'Arrow, puis d'Arrow-Kurz a été de poser le problème du taux d'actualisation en termes de politique optimale (par comparaison avec Marglin, Feldstein, ou encore Harberger et sa distinction entre le "Bureau d'Evaluation des Projets" et le "Ministère des Finances").

Nous avons indiqué que lorsque le financement et la répartition du produit des investissements publics sont déterminés de façon univoque (le seul degré de liberté étant le niveau de l'investissement public), ou sont indifférents (en un optimum), le coút d'opportunité du capital public, calculé à partir du coût d'opportunité du capital privé, fournit une approche simple et intuitive; il ne doit cependant pas être confondu avec le coût de rareté des fonds publics, qui tire son origine d'une contrainte sur le financement des investissements publics. 


\section{BIBLIOGRAPHIE}

[1] ATKINSON A., STIGLITZ J., 1977 "Lectures on Public Economics", à paraître.

[2] ARROW K.J., 1966 "Discounting and Public Investment Criteria" in Kneese and Smith (eds.) Water Research (Resources for the Future, Baltimore), pp $13-22$.

[3] ARROW K.J., 1977 "The Rate of Discount on Public Investments with Imperfect Capital Markets", Harvard D.P. 559.

[4] ARROW K.J., LIND R.C., 1970 "Uncertainty and the Evaluation of Public Investment Decisions", American Economic Review, Vol. 60.

[5] ARROW K.J., KURZ M., 1970 "Public Investment, the Rate of Return and Optimal Fiscal Policy", John Hopkins Press.

[6] BAUMOL W.J., 1968 "On the Social Rate of Discount", American Economic Review, Vol. 58.

[7] BAuMOL W.J., 1970 "Public Expenditures and Policy Analysis" HavermanMargolis.

[8] BOITEUX M., 1956 "Sur la gestion des monopoles publics astreints à l'équilibre budgétaire", Econometrica.

[9] BOITEUX M., 1969 "Note sur le taux d'actualisation", Revue d'Economie Politique.

[10] BRADFORD D.F., 1975 "Constraints on Government Investment Opportunities and the Choice of a Discount Rate", American Economic Review.

[11] DELORME R., 1976 "A propos du taux d'actualisation du Plan", Revue Economique, Janvier.

[12] DIAMOND P., 1968 "The Opportunity Cost of Public Investment : Comment", Quarterly Journal of Economics, Vol. 82.

[13] DIAMOND P., MIRRLEES J., 1971 "Optimal Taxation and Public Production, I and II", American Economic Review.

[14] DIAMOND P., 1973. "Taxation and Public Production in a Growth Setting", in Models of Economic Growth" (eds. J. Mirrlees and N. Stern).

[15] DREZE J., SANDMO A., 1971 "Discount Rate for Public Investment in Closed and Open Economies", Economica. 
[16] FELDSTEIN M.J., 1965 "The Derivation of Social Time Preference Rates", Kyklos, Vol. 18 .

[17] FELDSTEIN M.J., 1973 "Financing in the Evaluation of Public Expenditure", in "Essays in Honor of Richard Musgrave", W.L. Smith.

[18] GUESNERIE R., 1977 "On the Direction of Tax Reform", Journal of Public Economics.

[19] GUESNERIE R., TIROLE J., 1979 "Gradient Projection Algorithms for Problems in Public Finance : A Note", Papier de Discussion CEPREMAP, N 7907.

L20] GRJEBINE T., 1976 "Critique de la théorie de l'actualisation telle quelle a été employée en France", Revue d'Economie Politique, Mai/Juin.

[21] HARGERGER A., 1972 "Project Evaluation (collected papers)" Mac Millan.

[22] HIRSCHLEIFER J., 1961 "Comment on Eckstein's survey", in Buchanan (ed.)

"Public Finances : Needs, sources and utilisation" Princeton University Press.

[23] HIRSCHLEIFER J., 1965-66 "Investment Decisions under Uncertainty", Quarterly Journal of Economics, Vol. 79 et 80.

[24] KAY J.A., 1972 "Social Discount Rates", Journal of Public Economics.

[25] KOOPMANS J.C., 1965 "On the Concept of Optimal Economic Growth", in Study Week on "The Econometric Approach to Development Planning", pp 225-257, Amsterdam-North-Holland.

[26] MALINVAUD E., 1969 "Leçons de théorie microéconomique", Dunod.

[27] MARGLIN S.A., 1963a "The Social Rate of Discount and the Optimal Rate of Investment "Quarterly Journal of Economics.

1963b "Approaches to Dynamic Investment Planning".

1963c "The Opportunity Costs of Public Investment", Quarterly Journal of Economics.

[28] MISHAN, 1967 "Criteria for Public Investment : Some Simplifying Suggestions, Journal of Political Economy.

[29] NAOURI J.C., 1973 "Financement des investissements publics", Revue Economique, Juillet.

[30] PESTIAU P.M., 1974 "Optimal Taxation and Discount Rate for Public Investment in a Growth Setting", Journal of Public Economics. 
[31] PESTIAU P.M., POSSEN U., 1978 "Optimal Growth and Distribution Policies", Journal of Public Economics.

[32] PHELPS. E.S., SHELL K., 1969 "Public Debt, Taxation and Capital Intensiveness", Journal of Economic Theory.

[33] SEN A.K., 1967 "Isolation, Assurance and the Social Rate of Discount", Quarterly Journal of Economics.

[34] THEDIE J., 1974 "A Challenge to the Monopoly of the Rate of Discount in Investment Choices" in "The Economics of Public Services" (M.S. FeldsteinR.P. Inman, eds], Mac Milian Press, 1977.

[35] TIROLE J., 1978 "Essais sur le calcul économique public et sur le taux d'actualisation". Thèse pour le doctorat de 3ème cycle, Paris IXDauphine, UER Mathématiques de la Décision.

[36] TULLOCK G., 1964 "The Social Rate of Discount and the Optimal Rate of Investment. Comment", Quarterly Journal of Economics. 